\section{Tendência da prática de atividade física no lazer entre adultos no Brasil (2006-2016)}

\author{
Trends in leisure-time physical activity in \\ Brazilian adults (2006-2016)
}

\section{Tendencia de la práctica de actividad física durante el tiempo de ocio entre adultos en Brasil (2006-2016)}

Michele Santos da Cruz 1

Regina Tomie Ivata Bernal 2

Rafael Moreira Claro 3

doi: 10.1590/0102-311X00114817

\section{Resumo}

Este estudo objetiva analisar a tendência temporal da prática de atividade física no lazer entre adultos, nas capitais brasileiras e no Distrito Federal, entre 2006 e 2016. Foram utilizados dados do sistema de Vigilância de Fatores de Risco e Proteção para Doenças Crônicas por Inquérito Telefônico (VIGITEL), coletados pelo Ministério da Saúde no período entre 2006 e 2016 $(n=572.437)$. O VIGITEL entrevista anualmente mais de 50 mil indivíduos adultos (idade $\geq 18$ anos) que residem em domicílios com telefone fixo. As principais questões do VIGITEL de interesse deste estudo tratam da prática de atividade física no lazer. Estimou-se o percentual anual de realização de atividade física no lazer nos três meses que antecederam a entrevista, além daquele de prática em nível suficiente ( $\geq 150$ minutos/semana), disponível entre 2009 e 2016, para o conjunto da população, segundo sexo, idade e escolaridade. Verificou-se aumento $(p<0,05)$, tanto no percentual de prática de atividade física no lazer nos três meses que antecederam a entrevista (de 44,0 a 53,6\%, 0,97 pontos percentuais ( $p p) / a n o$ ) quanto no percentual de indivíduos que atingiram as recomendações de prática de atividade física: de 30,3 a 37,6\% (1,20pp/ano) entre 2009 a 2016. Os aumentos foram mais frequentes entre as mulheres, nos individuos nas menores faixas de idade e entre aqueles de maior escolaridade. Verificou-se aumento dos níveis de prática de atividade física na maioria das situações investigadas. Ainda que esse aumento tenha reduzido as diferenças entre homens e mulheres, foram acentuadas as diferenças entre jovens e pessoas mais velhas e entre aqueles nos niveis extremos de escolaridade.

Exercício; Doenças Não Transmissíveis; Adulto

\author{
Correspondência \\ M. S. Cruz \\ Rua São Carlos do Pinhal 79, apto. 114, São Paulo, SP \\ 01333-900, Brasil. \\ chele1213@gmail.com \\ 1 Faculdade de Saúde Pública, Universidade de São Paulo, São \\ Paulo, Brasil. \\ 2 Faculdade de Informática e Administração Paulista, São Paulo, \\ Brasil. \\ 3 Escola de Enfermagem, Universidade Federal de Minas Gerais, \\ Belo Horizonte, Brasil.
}




\section{Introdução}

As doenças crônicas não transmissíveis (DCNT) figuram entre os principais problemas de saúde pública, tanto em países desenvolvidos quanto naqueles em desenvolvimento ${ }^{1}$. No Brasil, as DCNT se tornaram a principal prioridade na área da saúde, visto que são responsáveis por $72 \%$ das mortes ocorridas no ano de 2007 2, valor 20\% superior à tendência internacional 3. De etiologia multifatorial, as DCNT compartilham fatores de risco modificáveis, tais como tabagismo, prática insuficiente de atividade física, alimentação inadequada e consumo abusivo de álcool 3,4. Uma vez controlados, esses fatores podem prevenir a maioria das DCNT ou minimizar seus prejuízos 3,4.

De fato, o papel da prática de atividade física na manutenção da saúde e da qualidade de vida dos indivíduos é amplamente reconhecido na literatura 5,6. A prática de atividade física em níveis suficientes mostra-se capaz de auxiliar na prevenção de DCNT ou atenuar seus efeitos em indivíduos em que essas doenças já se façam presentes 7 . Atualmente, o Ministério da Saúde adota para a população brasileira a recomendação, proferida pela Organização Mundial da Saúde (OMS), de prática de atividade física. Sugere-se prática mínima de 150 minutos por semana de atividade de intensidade moderada ou equivalente (75 minutos de atividade de intensidade vigorosa) ${ }^{8,9}$. Estimativas globais indicam que a prática insuficiente de atividade física seja responsável por cerca de 3,2 milhões de mortes ao ano $(5,5 \%)$, respondendo como causa, dentre outras, de $6 \%$ das doenças cardíacas, $7 \%$ dos casos de diabetes tipo II, 10\% de cânceres de mama e 10\% de cânceres de cólon 11.

Com isso, a Política Nacional de Promoção da Saúde (PNPS), aprovada no ano de 2006, elegeu a promoção, informação e educação em saúde como macroprioridades, com destaque para a importância da atividade física entre as estratégias de promoção da saúde no país 12. A partir desse marco, as estratégias adotadas nos últimos dez anos pelo Sistema Único de Saúde favoreceram a inserção de ações com ênfase no estímulo à prática de atividade física, como a criação do Núcleo de Apoio à Saúde da Família (Nasf) em 2008 (que inclui o profissional de educação física) 13 e a implementação do Programa Academia da Saúde, lançado em 2011 (com estrutura física e incentivo às atividades físicas ligadas à promoção da saúde) 14,15. Ainda que evidências iniciais indiquem a associação positiva entre essas ações e o nível de prática de atividade física na população, o monitoramento constante desse importante comportamento faz-se necessário, uma vez que uma parcela significativa da população declara realizar atividades físicas em nível aquém do ideal.

Monitorar os padrões de prática de atividade física e demais fatores de risco comportamentais na população emerge como um importante processo para a prevenção e o controle das DCNT 1. Entretanto, até o início dos anos 2000, o país não dispunha de dados qualificados para esse fim. Nesse contexto, a Secretaria de Vigilância em Saúde (SVS), órgão do Ministério da Saúde, em 2006, implantou o sistema de Vigilância de Fatores de Risco e Proteção para Doenças Crônicas por Inquérito Telefônico (VIGITEL), que se caracteriza por ser um sistema contínuo de monitoramento de fatores de risco e proteção das DCNT 16. O VIGITEL possibilita não apenas o monitoramento dos principais fatores de risco e proteção para DCNT no Brasil, como também fornece informações importantes para que se planejem políticas públicas de promoção e prevenção, além de se avaliarem as intervenções realizadas 17 . Atualmente os dados do VIGITEL são a principal fonte de informações para estudos de tendência da prática de atividade física entre adultos no país. Em estudo que avaliou a atividade física em quatro domínios (tempo livre, trabalho, em casa e no deslocamento) e também o comportamento sedentário (tempo assistindo à televisão), no período entre 2006 e 2009, contatou-se evolução positiva apenas quanto à atividade física no deslocamento (11,7 para 14,4\%), sem variações nos demais indicadores 18 . Em momento posterior, avaliou-se o mesmo conjunto de indicadores para o período entre $2006 \mathrm{e}$ 2012 19. Mudanças no questionário do VIGITEL em 2010 inviabilizaram que o indicador de prática de atividade física no lazer pudesse ser estimado segundo as recomendações da OMS. Entretanto, a utilização de indicador alternativo (atividade física $\geq 5$ dias/semana e $\geq 30$ minutos/dia) permitiu a análise de tendência com dados de todo período. Observou-se aumento no percentual de atividade física no lazer (de 12,8\% em 2006 para 14,9\% em 2012) 19. Resultado semelhante foi obtido também por meio de estudo que analisou dados do VIGITEL para o período entre 2009 e 2013 20. Nessa ocasião, observou-se aumento no percentual de ativos no tempo livre (de 29,9\% em 2009 a 33,8\% em 2013), redução no percentual de ativos no deslocamento para o trabalho/escola (de 17\% em 2009 a 12,1\% em 2013) e estabilidade nos demais indicadores 20 . Estudos semelhantes foram conduzidos também com foco 
específico para as capitais das regiões Sul 21, Norte 22 e Nordeste do Brasil 23 ou mesmo para o tipo datividade física de lazer praticado entre os anos 24 .

Com base nesse cenário, o presente estudo tem por objetivo atualizar a tendência temporal da prática de atividade física no lazer entre os adultos que residem nas capitais brasileiras e no Distrito Federal, entre 2006 e 2016.

\section{Métodos}

\section{Amostragem e coleta dos dados}

Serviram de base para este estudo dados coletados pelo VIGITEL entre os anos de 2006 e 2016. Os procedimentos de amostragem empregados pelo VIGITEL visam a obter, em cada uma das capitais dos estados brasileiros e no Distrito Federal, amostras probabilísticas da população de adultos residentes em domicílios servidos por, ao menos, uma linha telefônica fixa 9,16,25,26,27,28,29,30,31,32,33. No período entre 2006 e 2011, o sistema estabeleceu um tamanho amostral mínimo de 2 mil indivíduos com 18 ou mais anos de idade em cada cidade, com amostra anual média de cerca de 54 mil indivíduos. Excepcionalmente nos anos de 2012, 2013 e 2014, em função de dificuldades técnicas relacionadas à coleta dos dados, optou-se por reduzir o tamanho amostral mínimo para 1.600 indivíduos, retornando-se ao tamanho mínimo anteriormente definido nos anos de 2015 e 2016 16,25,26,27,28,29,30,31,32,33. Foi definido o tamanho amostral mínimo de aproximadamente 1.500 indivíduos em cada cidade, a fim de permitir estimar, com coeficiente de $95 \%$ de confiança e erro máximo de cerca de três pontos percentuais, a frequência de qualquer fator de risco na população adulta. Erros máximos de cerca de quatro pontos percentuais são esperados para estimativas específicas, segundo sexo, assumindo-se proporções semelhantes de homens e mulheres na amostra 34. Para os anos em que amostras mínimas de 2 mil indivíduos foram adotadas, o erro máximo nas estimativas sofre redução de 1 ponto percentual (pp), sem que a comparabilidade dos dados entre os anos seja afetada 34.

Em cada um dos anos de realização do inquérito, a etapa inicial da amostragem consistiu no sorteio de, no mínimo, 5 mil linhas telefônicas por cidade. Esse sorteio é realizado a partir do cadastro eletrônico de linhas residenciais fixas das principais empresas de telefonia fixa em operação no país. Em seguida, as linhas sorteadas em cada cidade são organizadas em réplicas de 200 linhas, reproduzindo a distribuição do cadastro original. A etapa seguinte do processo de amostragem consiste na confirmação da elegibilidade da linha sorteada e na seleção aleatória de um adulto ( $\geq 18$ anos) entre os residentes, realizada a partir de contato com o próprio número sorteado. Na Tabela 1, está exposto, para cada um dos sete segmentos do VIGITEL utilizados nesse estudo, o número total de linhas sorteadas e o número médio de entrevistas completadas por cidade 9,16,25,26,27,28,29,30,31,32,33.

O questionário do VIGITEL foi desenvolvido de modo a viabilizar a opção do sistema pela realização de entrevistas telefônicas feitas com o emprego de computadores (CATI), ou seja, entrevistas cujas perguntas são lidas na tela de um monitor de vídeo, ao passo que as respostas são registradas diretamente em meio eletrônico. Ainda que o questionário tenha sofrido mudanças durante o período estudado, as temáticas incluídas permaneceram constantes: características demográficas e socioeconômicas dos indivíduos (como idade, sexo e nível de escolaridade), indicadores do consumo alimentar, indicadores da prática de atividade física, peso e altura autorreferidos, consumo de cigarros e de bebidas alcoólicas, autoavaliação do estado de saúde, referência a diagnóstico médico de hipertensão arterial e diabetes, realização de exames para detecção precoce de câncer em mulheres e posse de plano de saúde ou convênio médico. Dentre as informações disponíveis no VIGITEL, são de interesse central deste estudo as referentes à prática de atividade física no lazer: "Nos últimos três meses, o(a) senhor(a) praticou algum tipo de exercício físico ou esporte? (sim/não)"; "Qual o tipo principal de exercício físico ou esporte que o(a) senhor(a) praticou? (caminhada/caminhada em esteira/corrida (cooper)/corrida em esteira/musculação/ginástica aeróbica (spinning, step, jump)/hidroginástica/ginástica em geral (alongamento, pilates, ioga)/natação/artes marciais e luta (jiu-jitsu, karatê, judô, boxe, muay thai, capoeira)/ bicicleta (inclui ergométrica)/futebol/futsal/basquetebol/voleibol/futevôlei/tênis/dança (balé, dança de salão, dança do ventre)"; "Quantos dias por semana o(a) senhor(a) costuma praticar exercício físico ou esporte? (1-2/3-4/5-6/todos os dias)"; "No dia em que o(a) senhor(a) pratica exercício ou esporte, 
Tabela 1

Número total de linhas sorteadas e número médio de entrevistas completadas, por ano, no conjunto das 26 capitais de estado e Distrito Federal. VIGITEL Brasil, 2006-2016.

\begin{tabular}{lcc}
\hline Ano do VIGITEL & Linhas sorteadas & Número de entrevistas \\
\hline 2006 & 135.000 & 54.369 \\
2007 & 138.600 & 54.251 \\
2008 & 106.000 & 54.353 \\
2009 & 135.200 & 54.367 \\
2010 & 153.000 & 54.339 \\
2011 & 135.000 & 54.144 \\
2012 & 135.000 & 45.448 \\
2013 & 135.000 & 52.929 \\
2014 & 101.200 & 40.853 \\
2015 & 135.000 & 54.174 \\
2016 & 189.000 & 53.210 \\
Total & $\mathbf{1 . 4 9 8 . 0 0 0}$ & $\mathbf{5 7 2 . 4 3 7}$ \\
\hline
\end{tabular}

VIGITEL: Vigilância de Fatores de Risco e Proteção para Doenças Crônicas por Inquérito Telefônico.

Fonte: VIGITEL Brasil 9,16,25,26,27,28,29,30,31,32,33.

quanto tempo dura a atividade? (menos de 10 minutos/entre 10 e 19 minutos/entre 20 e 29 minutos/ entre 30 e 39 minutos/entre 40 e 49 minutos/entre 50 e 59 minutos/60 minutos ou mais)".

Mais informações sobre a amostragem e a metodologia de coleta de dados do VIGITEL podem ser encontradas na publicação anual de resultados do sistema ${ }^{33}$.

\section{Organização e análise dos dados}

A partir das informações envolvendo intensidade, duração e frequência da prática de atividade física no VIGITEL, foram estabelecidos dois indicadores. O primeiro destinava-se a identificar a prática de atividade física no lazer, sem distinção quanto ao tipo, frequência ou duração, sendo definido a partir de resposta positiva à questão que abordava a prática de atividade física nos últimos 90 dias.

Já o segundo indicador destinava-se a identificar a prática de atividade física no lazer, em relação às recomendações internacionais. Foi considerada adequada prática de atividade física equivalente a um mínimo de 150 minutos semanais de atividade de intensidade moderada ou equivalente (75 minutos de atividade vigorosa) 8, a partir de combinação de respostas, permitindo atingir o limite mínimo recomendado nas questões, envolvendo tipo, frequência semanal e duração média de prática de atividade física. Esse indicador leva em consideração o compêndio de atividade física apresentado por Ainsworth et al. ${ }^{35}$, que classifica a intensidade das diferentes atividades de acordo com o dispêndio energético expresso em MET (Metabolic Equivalent of Task), equivalente metabólico que define as intensidades em leve (<3 METs), moderada (de 3 a 6 METs) e vigorosa (> 6 METs). Desse modo, dentre as atividades apresentadas no VIGITEL, estas são classificadas como de intensidade moderada: caminhada, caminhada em esteira, musculação, hidroginástica, ginástica em geral, natação, artes marciais, ciclismo e voleibol. Todas as demais são classificadas como de intensidade vigorosa 35 .

O primeiro indicador pôde ser calculado para todas as edições do VIGITEL utilizadas no presente estudo, ao passo que o segundo só pôde ser estimado para o período entre 2009 e 2016, devido a mudanças ocorridas no questionário (adotou-se maior número de categorias entre as opções de resposta para as questões que tratavam da frequência semanal e da duração de cada episódio de prática).

Inicialmente, a população de estudo foi descrita, em cada um dos anos, por meio de sua distribuição entre sexo (masculino e feminino), seis faixas de idade (18-24 anos/25-34 anos/35-44 anos/45-54 anos/55-64 anos e $\geq 65$ anos) e três faixas de escolaridade (0-8 anos/9-11 anos e $\geq 12$ anos de escolaridade). Em seguida, foi estimada a prevalência anual (para todos os anos em que se encontrava dis- 
ponível) de cada um dos indicadores de prática de atividade física para o conjunto total da população, segundo sexo, idade e escolaridade.

Modelos de regressão linear de Prais-Winsten (apropriados para utilização em análises de série temporal 36) foram empregados na análise da magnitude e significância estatística das variações temporais das situações acima descritas para todo o período estudado e, em seguida, segundo três intervalos de tempo (splines lineares, com dois nós para o período completo e um para os indicadores disponíveis desde 2009): 2006/2008, 2009/2012 e 2013/2016. Esses modelos tiveram como desfecho (variável dependente) o valor do indicador (por exemplo, o percentual de praticantes de atividade física no lazer) e, como variável explanatória, o ano do levantamento, expresso como variável contínua. Desse modo, o coeficiente de regressão do modelo (beta) indica a taxa média anual, expressa na unidade do desfecho (pp), de aumento ou diminuição do indicador no período. Foram consideradas significativas as variações temporais correspondentes a um coeficiente de regressão estatisticamente diferente de zero (valor de $\mathrm{p} \leq 0,05$ ). Analisou-se a variação no conjunto completo do período em que o indicador se fizesse disponível e, alternativamente, no período mais recente de seis anos (metade mais recente do período estudado e período próximo ao mínimo recomendado para análises de série temporal) 36 .

Foram utilizados fatores de ponderação do VIGITEL que visavam a corrigir a probabilidade desigual atestada na participação de alguns indivíduos da amostra, por residirem em domicílios com maior número de linhas telefônicas ou menor número de moradores. Esses fatores também permitiram a equiparação da distribuição da população estudada pelo VIGITEL - segundo sexo, idade e escolaridade - aquela identificada para a população adulta de cada uma das cidades, incluindo indivíduos com e sem telefone (a partir de dados do censo ou projeções intercensitárias, utilizando-se procedimento de pós-estratificação) 9 .

O aplicativo Stata versão 13.1 (https://www.stata.com) foi utilizado para organização e análise dos dados (StataCorp LP, College Station, Estados Unidos). O VIGITEL foi aprovado pela Comissão Nacional de Ética em Pesquisa (CONEP) do Conselho Nacional de Saúde (no 355.590).

\section{Resultados}

No período entre 2006 e 2016, um total de 572.437 indivíduos foi entrevistado pelo VIGITEL. Essa população foi composta, em sua maioria, por mulheres (cerca de $54 \%$ em qualquer um dos anos). Nesse mesmo período, é possível observar mudanças significativas na distribuição de idade e escolaridade da população adulta que residia nas capitais dos estados brasileiros 30 . Enquanto a proporção de indivíduos com idade entre 18 e 24 anos diminuiu, em média, 0,38pp/ano ( $\mathrm{p}<0,01)$, a proporção de indivíduos nas faixas de idade acima de 45 anos aumentou significativamente: $0,17 \mathrm{pp} /$ ano para a faixa de 45-54 anos; 0,24pp/ano para a faixa de 55-64 anos e 0,14pp/ano para faixa 65 anos e mais ( $\mathrm{p}<0,01$ ). De modo semelhante, enquanto a proporção de indivíduos na faixa inferior de escolaridade, zero a oito anos completos de estudo, diminuiu 1,31pp/ano ( $\mathrm{p}<0,001)$, a de indivíduos com escolaridade superior a nove anos completos de estudo aumentou significativamente: 0,36pp/ano para a faixa de 9-11 anos e 0,92pp/ano para a faixa 12 anos ou mais $(\mathrm{p}<0,01)$ (Tabela 2$)$.

No conjunto completo do período estudado, a frequência de indivíduos que alegaram ter realizado atividade física no lazer nos três meses que antecederam a entrevista aumentou de $44 \%$ para $53,6 \%$, com aumento médio anual de $0,97 \mathrm{pp}(\mathrm{p}<0,05)$. Incremento de maior magnitude foi observado entre mulheres - de 35\%-46,4\% (em média 1,16pp/ano, p <0,05) - e entre adultos jovens (indivíduos com idade entre 18 e 34 anos), com variação de 56\%-67,5\% (em média 1,19pp/ano, p < 0,05) para aqueles entre 18 e 24 anos, e de 48,6\%-60,9\% (em média 1,31pp/ano, p < 0,05) para aqueles entre 25 e 34 anos. De forma alternativa, a análise por períodos evidencia a aceleração do crescimento do indicador no período mais recente, com taxas de incremento sistematicamente superiores àquelas observadas no período completo do estudo (Tabela 3).

De modo semelhante, verificou-se também aumento na porcentagem de indivíduos que atingiram o volume recomendado de atividade física (150 minutos/semanais de atividade moderada ou equivalente) com prática de atividade física no lazer (Figura 1). Entre os anos de 2009 e 2016, a frequência de indivíduos nessa condição aumentou de 30,3 a 37,6\% (1,20pp/ano, p < 0,05). Uma vez mais, as maiores 


\section{Tabela 2}

Distribuição da população adulta ( $\geq 18$ anos) das capitais dos 26 estados brasileiros e do Distrito Federal, segundo sexo, idade e escolaridade, por ano. VIGITEL Brasil, 2006-2016.

\begin{tabular}{|c|c|c|c|c|c|c|c|c|c|c|c|c|c|}
\hline Variáveis & 2006 & 2007 & 2008 & 2009 & 2010 & 2011 & 2012 & 2013 & 2014 & 2015 & 2016 & $\begin{array}{l}\text { Incremento médio * (pp/ano) } \\
2006-2016\end{array}$ & Valor de $p$ ** \\
\hline \multicolumn{14}{|l|}{ Sexo } \\
\hline Masculino & 46,1 & 46,2 & 46,1 & 46,1 & 46,1 & 46,1 & 46,1 & 46,1 & 46,1 & 46,0 & 46,0 & $-0,01$ & 0,011 \\
\hline Feminino & 53,9 & 53,8 & 53,9 & 53,9 & 53,9 & 53,9 & 53,9 & 53,9 & 53,9 & 54,0 & 54,0 & 0,010 & 0,011 \\
\hline \multicolumn{14}{|l|}{ Idade (anos) } \\
\hline $18-24$ & 18,9 & 18,2 & 17,9 & 17,2 & 17,1 & 16,7 & 16,4 & 15,9 & 15,6 & 15,2 & 14,8 & $-0,384$ & $<0,001$ \\
\hline $25-34$ & 25,4 & 25,4 & 25,4 & 25,5 & 25,4 & 25,4 & 25,2 & 25,3 & 25,3 & 25,2 & 25,2 & $-0,022$ & 0,004 \\
\hline $35-44$ & 20,6 & 20,5 & 20,4 & 20,3 & 20,1 & 20,0 & 19,9 & 19,7 & 19,6 & 19,4 & 19,3 & $-0,136$ & $<0,001$ \\
\hline $45-54$ & 15,8 & 15,9 & 16,1 & 16,3 & 16,4 & 16,6 & 16,8 & 16,9 & 17,1 & 17,3 & 17,4 & 0,165 & $<0,001$ \\
\hline $55-64$ & 10,0 & 10,2 & 10,4 & 10,7 & 10,9 & 11,1 & 11,4 & 11,6 & 11,8 & 12,1 & 12,3 & 0,236 & $<0,001$ \\
\hline 65 e mais & 9,4 & 9,8 & 9,8 & 10,0 & 10,1 & 10,2 & 10,4 & 10,5 & 10,6 & 10,8 & 10,9 & 0,141 & $<0,001$ \\
\hline \multicolumn{14}{|c|}{ Escolaridade (anos) } \\
\hline $0-8$ & 45,5 & 45,0 & 43,7 & 42,0 & 40,6 & 38,8 & 36,8 & 36,6 & 35,9 & 34,6 & 32,5 & $-1,312$ & $<0,001$ \\
\hline $9-11$ & 33,3 & 35,1 & 34,7 & 35,8 & 35,8 & 36,7 & 38,5 & 37,5 & 38,1 & 38,1 & 35,9 & 0,363 & 0,007 \\
\hline 12 e mais & 21,2 & 19,8 & 21,6 & 22,2 & 23,5 & 24,5 & 24,7 & 25,9 & 25,9 & 27,3 & 31,6 & 0,924 & $<0,001$ \\
\hline
\end{tabular}

VIGITEL: Vigilância de Fatores de Risco e Proteção para Doenças Crônicas por Inquérito Telefônico.

* Correspondente ao coeficiente da regressão de Prais-Winsten do valor do indicador sobre o ano do levantamento;

** O significado estatístico da tendência do indicador no período foi avaliado por meio de modelo de regressão linear, tendo como desfecho (variável dependente) o valor do indicador (por exemplo, o percentual de indivíduos ativos no lazer no ano) e, como variável explanatória, o ano do levantamento, expresso como variável contínua.

magnitudes de aumento foram observadas entre as mulheres (de 22,2\%-29,9\%, em média 1,38pp/ano, $\mathrm{p}<0,01$ ), indivíduos nas faixas de idade de 25-34 anos (de 34,3\%-46\%, em média 1,80pp/ano, p < 0,01) e na faixa superior de escolaridade (de 42\%-47,9\%, em média 1,26pp/ano, p < 0,01). Uma vez mais, observaram-se, de forma geral, maiores magnitudes de aumento no terço final do período estudado (2013-2016), exceto entre as mulheres e indivíduos com idade entre 18 e 24 anos (Tabela 4).

\section{Discussão}

A partir da coleta sistemática de dados realizada pelo VIGITEL, entre os anos de 2006 e 2016, em uma amostra de mais de 570 mil indivíduos, representativa da população adulta das 26 capitais dos estados brasileiros e do Distrito Federal, foi possível analisar a evolução da prática de atividade física no lazer no país. Inicialmente, no conjunto completo do período estudado (2006-2016), verificou-se aumento significativo da proporção de indivíduos que alegaram ter praticado atividade física no lazer nos três meses que antecederam a entrevista, de $44 \%$ a 53,6\% (0,97pp/ano). Em consequência, no período de 2009-2016, observou-se também aumento no percentual de indivíduos que atingiram o nível mínimo recomendado de prática de atividade física (150 minutos/semana de atividade de intensidade moderada ou equivalente) no lazer, de 30,3\%-37,6\% (1,20pp/ano). Esse resultado fornece importante atualização para estimativas divulgadas em outras publicações destinadas a avaliar tendências da prática de atividade física no lazer no país 18,19,37.

$\mathrm{O}$ aumento verificado em nosso estudo para o percentual de indivíduos que alegaram ter praticado atividade física nos três meses que antecederam a entrevista apresenta a real dimensão do aumento da prática de atividade física no lazer entre adultos nas capitais de estado e no Distrito Federal. Ainda que parte desses indivíduos não possua volume suficiente de atividade física para atingir as recomendações da OMS, desenvolver o hábito de praticar atividades físicas - mesmo que com fre- 
Tabela 3

Evolução anual da frequência de indivíduos que declararam ter praticado atividade física no lazer nos três meses que antecederam a entrevista na população adulta ( $\geq 18$ anos) das capitais dos 26 estados brasileiros e do Distrito Federal, segundo sexo, idade e anos de escolaridade, por ano. VIGITEL Brasil, 2006-2016.

\begin{tabular}{|c|c|c|c|c|c|c|c|c|c|c|c|c|c|c|c|}
\hline \multirow[t]{2}{*}{ Variáveis } & \multirow[t]{2}{*}{2006} & \multirow[t]{2}{*}{2007} & \multirow[t]{2}{*}{2008} & \multirow[t]{2}{*}{2009} & \multirow[t]{2}{*}{2010} & \multirow[t]{2}{*}{2011} & \multirow[t]{2}{*}{2012} & \multirow[t]{2}{*}{2013} & \multirow[t]{2}{*}{2014} & \multirow[t]{2}{*}{2015} & \multirow[t]{2}{*}{2016} & \multicolumn{4}{|c|}{ Incremento médio * (pp/ano) } \\
\hline & & & & & & & & & & & & 2006-2008 & 2009-2012 & 2013-2016 & 2006-2016 \\
\hline \multicolumn{16}{|l|}{ Sexo } \\
\hline Masculino & 54,5 & 53,0 & 52,1 & 53,5 & 52,9 & 55,1 & 55,4 & 55,0 & 56,9 & 58,7 & 62,0 & $-0,94$ & 0,55 & $1,56 * \star$ & $0,74 * *$ \\
\hline Feminino & 35,0 & 36,4 & 35,3 & 34,6 & 35,3 & 37,2 & 39,9 & 40,5 & 43,2 & 44,8 & 46,4 & $-0,52$ & 1,05 * & $1,98 * \star$ & $1,16 * \star$ \\
\hline \multicolumn{16}{|l|}{ Idade (anos) } \\
\hline $18-24$ & 56,0 & 56,1 & 56,4 & 57,0 & 57,7 & 57,6 & 61,2 & 62,3 & 65,0 & 64,9 & 67,5 & $-0,11$ & $1,10 * \star$ & $1,81 \star \star \star$ & $1,19 * *$ \\
\hline $25-34$ & 48,6 & 47,1 & 44,7 & 47,4 & 47,0 & 49,7 & 52,2 & 52,8 & 55,0 & 57,7 & 60,9 & $-1,86 * *$ & $1,53 * \star$ & $2,23 * \star$ & $1,31 * *$ \\
\hline $35-44$ & 39,9 & 40,8 & 38,8 & 37,4 & 37,9 & 40,4 & 43,3 & 43,3 & 45,6 & 49,1 & 51,3 & $-1,43$ & $0,91 * \star$ & $2,42 * \star$ & 1,14 ** \\
\hline $45-54$ & 37,2 & 36,0 & 37,6 & 37,7 & 37,5 & 39,4 & 39,4 & 40,1 & 43,8 & 44,2 & 46,6 & 0,31 & $0,54 * *$ & $1,79 * \star$ & $0,96 * *$ \\
\hline $55-64$ & 40,1 & 41,5 & 39,5 & 38,0 & 38,8 & 40,1 & 40,1 & 40,3 & 43,1 & 44,3 & 47,3 & $-0,95$ & 0,06 & $1,80 * *$ & 0,68 \\
\hline 65 e mais & 31,8 & 36,9 & 36,4 & 36,2 & 35,6 & 40,5 & 39,4 & 36,7 & 37,2 & 39,8 & 40,5 & 1,79 & 0,41 & 0,34 & $0,56 * *$ \\
\hline \multicolumn{16}{|c|}{ Escolaridade (anos) } \\
\hline $0-8$ & 32,4 & 32,6 & 32,3 & 30,5 & 30,4 & 33,4 & 33,5 & 33,9 & 35,6 & 36,5 & 38,5 & $-0,77$ & 0,42 & 1,32 ** & $0,60 * *$ \\
\hline $9-11$ & 49,3 & 50,0 & 47,3 & 48,4 & 47,6 & 48,5 & 50,2 & 49,8 & 52,1 & 53,3 & 55,2 & $-1,27$ & 0,35 & $1,46 * \star$ & $0,58 * *$ \\
\hline 12 e mais & 60,4 & 59,7 & 57,9 & 59,4 & 59,4 & 59,9 & 62,2 & 62,1 & 64,9 & 66,9 & 67,4 & $-1,21 * *$ & 0,74 ** & 1,73 ** & 0,76 ** \\
\hline Total & 44,0 & 44,1 & 43,1 & 43,3 & 43,4 & 45,4 & 47,0 & 47,2 & 49,5 & 51,2 & 53,6 & $-0,81$ & $0,83 * *$ & $1,79 * \star$ & $0,97 * \star$ \\
\hline
\end{tabular}

VIGITEL: Vigilância de Fatores de Risco e Proteção para Doenças Crônicas por Inquérito Telefônico.

Nota: O significado estatístico da tendência do indicador no período foi avaliado por meio de modelo de regressão linear, tendo como desfecho (variável dependente) o valor do indicador (por exemplo, o percentual de indivíduos ativos no lazer no ano) e, como variável explanatória, o ano do levantamento, expresso como variável contínua.

* Correspondente ao coeficiente da regressão de Prais-Winsten, utilizando splines lineares com dois nós (2006/2008, 2009/2012 e 2013/2016), do valor do indicador sobre o ano do levantamento;

** $p<0,05$.

quência insuficiente - já pode ser visto como um estágio inicial de adoção de um estilo de vida mais saudável 38,39. Entretanto, cerca de metade da população permanece sem realizar nenhum tipo da atividade física no lazer, valor considerado preocupante.

O percentual de indivíduos que declararam praticar o nível recomendado de atividade física em atividades de lazer subiu, entre 2009 e 2016, tanto entre homens (0,96pp/ano) quanto entre mulheres (1,38pp/ano). O maior aumento verificado entre as mulheres é positivo e age no sentido de reduzir os menores percentuais apresentados por elas quando comparadas aos homens no início do período estudado, confirmando a tendência de diminuição da desigualdade entre os sexos, previamente observada em estudo nacional que apresentou a tendência da prática de atividade física de 2006-2012 19 e em estudo de revisão sistemática de literatura 40. Com isso, a diferença entre os sexos que, em 2009 , era de 17,5 pp (22,2\% para as mulheres e $39,7 \%$ para os homens) caiu para $16,7 \mathrm{pp}$ (29,9\% para as mulheres e $46,6 \%$ para os homens), ainda que os homens permaneçam com percentual de prática muito superior ao das mulheres.

Por outro lado, as tendências observadas na última década aumentaram as diferenças observadas no início do período estudado quanto à faixa de idade e ao nível de escolaridade. Destacou-se nesse estudo o baixo aumento na prática de atividade física no tempo de lazer entre os indivíduos com 65 anos de idade ou mais, contrastando com os altos índices de aumento verificados entre os adultos jovens (indivíduos com menos de 35 anos de idade). Ainda que atividades físicas devam ser praticadas por indivíduos de todas as idades, elas são especialmente importantes para os indivíduos idosos, por agirem tanto como fator de proteção para DCNT entre indivíduos saudáveis 41,42,43, como também por sua associação a prognósticos mais favoráveis entre indivíduos enfermos 44,45,46. No mesmo sen- 


\section{Figura 1}

Evolução anual do percentual de adultos ( $\geq 18$ anos) que praticaram atividades físicas no lazer equivalentes a, pelo menos, 150 minutos de atividade de intensidade moderada por semana nas capitais dos 26 estados brasileiros e do Distrito Federal, segundo sexo. VIGITEL Brasil, $2009-2016$.

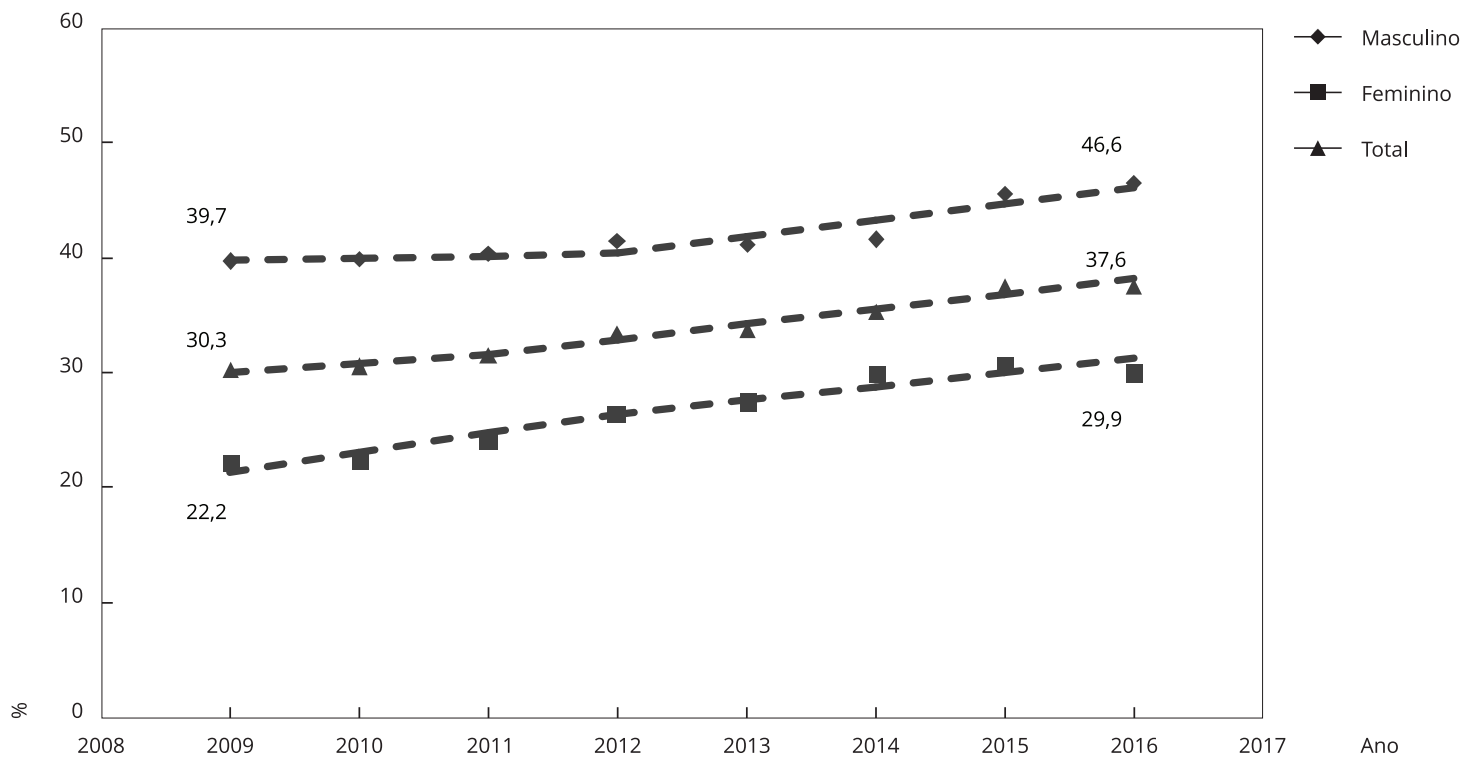

VIGITEL: Vigilância de Fatores de Risco e Proteção para Doenças Crônicas por Inquérito Telefônico.

Nota: linhas de tendência estimadas por meio de modelo de regressão de Prais-Winsten, utilizando splines lineares com um nó (2009/2012 e

2013/2016). Valores de R2 para os modelos: masculino =0,94; feminino =0,96; total =0,99.

tido, observou-se aumento na diferença da prevalência de prática em indivíduos nos níveis extremos de escolaridade, indicando novamente que a população do país segue trajetória semelhante àquela traçada por populações de países desenvolvidos. Estudo de tendência na prática de atividade física no Canadá, no período de 1981-2000, realizado junto à população adulta, já indicava que grupos populacionais com maiores níveis de escolaridade apresentavam prevalência mais elevada de prática quando comparados àqueles de nível de escolaridade mais baixo 47 . Ainda que os resultados do presente estudo evidenciem aumento em todas as categorias de escolaridade entre 2009 e 2016 (na faixa de 0-8 anos de estudos houve aumento de 0,79pp/ano no período, e na de 9-11 anos aumento de 0,86pp/ ano), é entre aqueles indivíduos com doze anos ou mais de estudo que são observados os aumentos de maior magnitude (1,26pp/ano).

As análises realizadas no presente estudo são incapazes de indicar as causas da evolução positiva verificada. Ainda que essa possa ser, ao menos em parte, devida a um amplo conjunto de ações desenvolvidas no âmbito do Sistema Único de Saúde (SUS) objetivando a promoção de saúde e a prevenção de doenças ${ }^{48}$, a ausência de estudos que comprovem a efetividade dessas ações ou mesmo sua consistência com nossos resultados impede que parcela significativa da melhora observada seja atribuída a elas. A caminhada em grupo é, tradicionalmente, a atividade mais presente nas unidades de saúde do país 49 , ainda que outras atividades tenham sido gradativamente aumentadas nos últimos anos em termos de oferta - tais como Lia-Gong, Tai chi chuan e, até mesmo, musculação 49 . Ao mesmo tempo, a criação dos Nasf 13 possibilitou o ingresso de um contingente expressivo de profissionais de Educação Física no SUS, ao mesmo tempo em que iniciativas como os Programas Academia da Saúde 14,15,50 e Academias da Terceira Idade 51 têm buscado ampliar a oferta de locais qualificados para a prática de atividade física, especialmente em vizinhanças de menor nível de renda e desenvolvimento social 14,15,51. Ainda que essas mudanças se configurem como iniciativas positivas 52, são claramente insuficientes na explicação da evolução observada no percentual de atividade física da população. 


\section{Tabela 4}

Evolução anual do percentual de adultos ( $\geq 18$ anos) que praticaram atividades físicas no lazer equivalentes a, pelo menos, 150 minutos de atividade de intensidade moderada por semana nas capitais dos 26 estados brasileiros e do Distrito Federal, segundo sexo, idade e escolaridade, por ano. VIGITEL Brasil, 2009-2016.

\begin{tabular}{|c|c|c|c|c|c|c|c|c|c|c|c|}
\hline \multirow[t]{2}{*}{ Variáveis } & \multirow[t]{2}{*}{2009} & \multirow[t]{2}{*}{2010} & \multirow[t]{2}{*}{2011} & \multirow[t]{2}{*}{2012} & \multirow[t]{2}{*}{2013} & \multirow[t]{2}{*}{2014} & \multirow[t]{2}{*}{2015} & \multirow[t]{2}{*}{2016} & \multicolumn{3}{|c|}{ Incremento médio * (pp/ano) } \\
\hline & & & & & & & & & 2009-2012 & 2013-2016 & 2009-2016 \\
\hline \multicolumn{12}{|l|}{ Sexo } \\
\hline Masculino & 39,7 & 40,0 & 40,4 & 41,5 & 41,2 & 41,6 & 45,6 & 46,6 & 0,22 & 1,43 ** & 0,96 ** \\
\hline Feminino & 22,2 & 22,4 & 24,1 & 26,5 & 27,4 & 30,0 & 30,8 & 29,9 & 1,68 * & $1,20 * *$ & $1,38 * \star$ \\
\hline \multicolumn{12}{|l|}{ Idade (anos) } \\
\hline $18-24$ & 43,2 & 44,0 & 44,4 & 47,6 & 49,7 & 50,0 & 51,4 & 52,2 & 1,60 * & 1,29 ** & $1,41 * \star$ \\
\hline $25-34$ & 34,3 & 34,9 & 36,0 & 39,1 & 39,3 & 41,5 & 45,2 & 46,0 & 1,35 * & 2,13 ** & 1,80 ** \\
\hline $35-44$ & 25,6 & 26,6 & 27,5 & 31,0 & 29,6 & 31,2 & 36,4 & 35,7 & 1,22 * & $1,80 * *$ & $1,56 \star \star$ \\
\hline $45-54$ & 24,8 & 24,6 & 26,5 & 25,8 & 27,3 & 30,1 & 30,5 & 30,4 & 0,66 & 1,24 ** & 0,98 ** \\
\hline $55-64$ & 24,3 & 24,5 & 25,5 & 25,2 & 26,6 & 28,4 & 29,1 & 29,7 & 0,45 * & $1,14 * \star$ & $0,84 * \star$ \\
\hline 65 e mais & 22,6 & 20,7 & 22,5 & 23,6 & 22,3 & 22,8 & 23,5 & 22,3 & 0,53 & $-0,03$ & 0,18 \\
\hline \multicolumn{12}{|c|}{ Escolaridade (anos) } \\
\hline $0-8$ & 19,9 & 20,1 & 21,2 & 21,6 & 22,0 & 22,9 & 25,4 & 24,5 & 0,53 * & $1,00 * *$ & $0,79 * \star$ \\
\hline $9-11$ & 35,2 & 35,0 & 35,3 & 37,1 & 37,2 & 38,5 & 40,1 & 40,4 & 0,56 * & 1,10 ** & 0,86 ** \\
\hline 12 e mais & 42,0 & 41,7 & 42,5 & 45,4 & 45,4 & 47,8 & 49,6 & 47,9 & $1,27 *$ & $1,26 * \star$ & $1,26 * \star$ \\
\hline Total & 30,3 & 30,5 & 31,6 & 33,5 & 33,8 & 35,3 & 37,6 & 37,6 & 1,01 * & 1,353 ** & 1,20 ** \\
\hline
\end{tabular}

VIGITEL: Vigilância de Fatores de Risco e Proteção para Doenças Crônicas por Inquérito Telefônico.

Nota: o significado estatístico da tendência do indicador no período foi avaliado por meio de modelo de regressão linear, tendo como desfecho (variável dependente) o valor do indicador (por exemplo, o percentual de indivíduos ativos no lazer no ano) e, como variável explanatória, o ano do levantamento, expresso como variável contínua.

* Correspondente ao coeficiente da regressão de Prais-Winsten, utilizando splines lineares com um nó (2009/2012 e 2013/2016), do valor do indicador sobre o ano do levantamento;

** $\mathrm{p}<0,05$.

Aumentos de maior magnitude foram observados entre adultos jovens (com idade entre 18 e 34 anos) e entre indivíduos na faixa superior de escolaridade, perfil muito distinto do observado nas iniciativas vinculadas ao SUS 53 .

Algumas limitações devem ser observadas quanto aos resultados de nosso estudo. Por se apoiar em entrevistas telefônicas, o VIGITEL não é capaz de medir diretamente a prática de atividade no lazer realizada pela população, baseando-se na condição declarada pelos entrevistados. Essa situação pode resultar na sub ou superestimação da prevalência real na prática de atividade física no lazer 28,54. Ainda assim, acredita-se que essa metodologia não afete intensamente os achados do presente estudo. Informações autorreferidas são frequentemente utilizadas em grandes inquéritos sobre condições de saúde e estilo de vida 55,56,57, devido à simplicidade e ao baixo custo de sua obtenção junto a grandes amostras populacionais 58,59,60. Além disso, a delimitação da amostra do VIGITEL somente aos indivíduos que residem em domicílios com telefone fixo representa um risco potencial à representatividade da amostra (uma vez que reduziria a participação de determinados grupos populacionais no estudo, com adultos jovens e indivíduos de baixa renda). Essa questão é minimizada pelo uso de fatores de ponderação dos dados, os quais buscam igualar as características demográficas da amostra do VIGITEL às características da população total, segundo dados do censo do Instituto Brasileiro de Geografia e Estatística e projeções oficiais para a população brasileira 9. Além disso, a metodologia utilizada para construção dos fatores de ponderação do VIGITEL foi inteiramente atualizada no ano de 2012 30, possibilitando, entre outros, incorporar mudanças demográficas ocorridas na população estudada nas estimativas anuais do sistema 30.

Mesmo diante do desafio atual de desenvolver programas e intervenções que promovam atividade física para o acesso de todos os grupos populacionais, a ação de explorar, fortalecer e ampliar 
os programas já existentes no sistema público pode configurar-se como importante alternativa na manutenção e promoção da atividade física, que deve ser potencializada e associada a orientações quanto aos benefícios da adoção de estilos de vida ativos e suas possibilidades (envolvendo o uso dos deslocamentos ativos). A elaboração de guias de práticas de atividades físicas para a população brasileira, de acordo com seus grupos populacionais, apresentando de forma clara e objetiva os benefícios da atividades física para a saúde também pode apresentar-se como ferramenta que motive a prática regular consciente. Destaca-se, ainda, que essas recomendações podem ser incorporadas à atuação dos atuais profissionais das unidades básicas de saúde e centros de saúde, expandindo ações já realizadas nesse nível da atenção à saúde no país.

No presente estudo, embora tenha sido identificado aumento nos níveis gerais de prática de atividade física, destaca-se que ainda é baixo o percentual de pessoas que atingem a recomendação diária (menos de 40\%). Essa situação evidencia a necessidade de que esforços políticos e sociais sejam mantidos, no sentido de promover a ampliação de espaços e investir em estruturas que permitam maior acesso da população às condições adequadas de prática de atividade física regular, para que esse percentual se mantenha potencialmente maior a cada ano.

\section{Conclusão}

Observou-se aumento na prevalência de prática de atividade física no lazer entre adultos nas capitais de estados do país e no Distrito Federal. Esse aumento foi especialmente relevante entre as mulheres, os adultos jovens e aqueles indivíduos com maior nível de escolaridade. Ainda que, de um lado, essa tendência tenha reduzido as diferenças observadas entre os sexos, por outro lado, acentuou as diferenças entre indivíduos mais jovens e mais velhos e entre aqueles nos níveis extremos de escolaridade.

\section{Colaboradores}

M. S. Cruz participou do delineamento do estudo, da análise e interpretação dos dados, da redação do manuscrito e da revisão. R. T. I. Bernal contribuiu com a interpretação dos dados, com a redação final do manuscrito e como a revisão. R. M. Claro participou do delineamento do estudo, da análise e interpretação dos dados, da redação do manuscrito e da revisão.

\section{Agradecimentos}

Os autores agradecem à Coordenação de Aperfeiçoamento de Pessoal de Nível Superior (Capes) pelo financiamento de bolsa de estudo.

\section{Referências}

1. World Health Organization; National Institute on Aging; National Institutes of Health. Global health and aging. s.l.: World Health Organization/National Institute on Aging/National Institutes of Health; 2011.

2. Schmidt MI, Duncan BB, Silva GA, Menezes AM, Monteiro CA, Barreto SM, et al. Doenças crônicas não transmissíveis no Brasil: carga e desafios atuais. Lancet 2011; 377:1949-61.

3. World Health Organization. Preventing chronic diseases: a vital investment. Geneva: World Health Organization; 2005.

4. Malta DC, Cezario AC, Moura L, Morais Neto OL, Silva Junior JB. A construção da vigilância e prevenção das doenças crônicas não transmissíveis no contexto do Sistema Único de Saúde. Epidemiol Serv Saúde 2006; 15:47-65.

5. Esteban C, Quintana JM, Aburto M, Moraza J, Egurrola M, Pérez-Izquierdo J, et al. Impact of changes in physical activity on health-related quality of life among patients with COPD. Eur Respir J 2010; 36:292-300.

6. Awick E, Wójcicki TR, Olson EA, Fanning J, Chung HD, Zuniga K, et al. Differential exercise effects on quality of life and health-related quality of life in older adults: a randomized controlled trial. Qual Life Res 2015; 24:455-62. 
7. Organização Pan-Americana da Saúde. Doenças crônico-degenerativas e obesidade: estratégia mundial sobre a alimentação saudável, atividade física e saúde. Brasília: Organização Pan-Americana da Saúde; 2003.

8. World Health Organization. Global recommendations on physical activity for health. Geneva: World Health Organization; 2010.

9. Departamento de Vigilância de Doenças e Agravos não transmissíveis e Promoção da Saúde, Secretaria de Vigilância em Saúde, Ministério da Saúde. Vigitel Brasil 2014: vigilância de fatores de risco e proteção para doenças crônicas por inquérito telefônico. Brasília: Ministério da Saúde; 2015.

10. World Health Organization. Global health risks: mortality and burden of disease attributable to selected major risks. Geneva: World Health Organization; 2009.

11. Lee MI, Shiroma EJ, Lobelo F, Puska P, Blair SN, Katzmarzyk PT. Effect of physical inactivity on major non-communicable diseases worldwide: an analysis of burden of disease and life expectancy. Lancet 2012; 6736:61031-9.

12. Secretaria de Atenção à Saúde, Secretaria de Vigilância em Saúde, Ministério da Saúde. Política Nacional de Promoção da Saúde. Brasília: Ministério da Saúde; 2006.

13. Ministério da Saúde. Portaria no 154, de 28 de janeiro de 2008. Cria os Núcleos de Apoio à Saúde da Família - NASF. Diário Oficial da União 2008; 4 mar.

14. Ministério da Saúde. Portaria no 719 , de 7 de abril de 2011. Institui o Programa Academia da Saúde no âmbito do Sistema Único de Saúde. Diário Oficial da União 2011; 8 abr.

15. Ministério da Saúde. Academia da saúde. Cartilha informativa. Brasília: Ministério da Saúde; 2014

16. Secretaria de Gestão Estratégica e Participativa, Secretaria de Vigilância em Saúde, Ministério da Saúde. Vigitel Brasil 2006: vigilância de fatores de risco e proteção para doenças crônicas por inquérito telefônico. Brasília: Ministério da Saúde; 2007. (Série G. Estatística e Informação em Saúde).

17. Departamento de Análise de Situação de Saúde, Secretaria de Vigilância em Saúde, Ministério da Saúde. Plano de ações estratégicas para o enfrentamento das doenças crônicas não transmissíveis (DCNT) no Brasil 2011-2022. Brasília: Ministério da Saúde; 2011. (Série B. Textos Básicos de Saúde).

18. Hallal PC, Knuth AG, Reis RS, Rombaldi AJ, Malta DC, Iser BPM, et al. Time trends of physical activity in Brazil (2006-2009). Rev Bras Epidemiol 2011; 14 Suppl 1:53-60.

19. Mielke GI, Hallal PC, Malta DC, Lee IM. Time trends of physical activity and television viewing time in Brazil: 2006-2012. Int J Behav Nutr Phys Act 2014; 11:101.

20. Malta DC, Andrade SSA, Santos MAS, Rodrigues GBA, Mielke GI. Tendências dos indicadores de atividade física em adultos, conjunto de capitais do Brasil 2006-2013. Rev Bras Ativ Fís Saúde 2015; 20:141-51.
21. Rech C, Reis R, Hino A, De Sá G, Stopa S, Malta $\mathrm{D}$. Tendências temporais de atividade física e comportamento sedentário nas capitais da Região Sul do Brasil: 2006-2013. Rev Bras Ativ Fís Saúde 2015; 20:47-56.

22. Mielke G, Costa DJS, Stopa SR, Campos MO, Pureza DY, Silva MMA. Tendência temporal de indicadores da prática de atividade física e comportamento sedentário nas capitais da Região Norte do Brasil: 2006-2013. Rev Bras Ativ Fís Saúde 2015; 20:130-40.

23. Tassitano R, Mielke G, Carvalho W, Oliveira M, Malta D. Tendência temporal de indicadores da prática de atividade física e comportamento sedentário nas capitais da Região Nordeste do Brasil: 2006-2013. Rev Bras Ativ Fís Saúde 2015; 20:152-64.

24. Sa TH, Garcia LMT, Claro RM. Frequency, distribution and time trends of types of leisure-time physical activity in Brazil, 20062012. Int J Public Health 2014; 59:975-82.

25. Secretaria de Gestão Estratégica e Participativa, Secretaria de Vigilância em Saúde, Ministério da Saúde. Vigitel Brasil 2007: vigilância de fatores de risco e proteção para doenças crônicas por inquérito telefônico. Brasília: Ministério da Saúde; 2009. (Série G. Estatística e Informação em Saúde).

26. Secretaria de Gestão Estratégica e Participativa, Secretaria de Vigilância em Saúde, Ministério da Saúde. Vigitel Brasil 2008: vigilância de fatores de risco e proteção para doenças crônicas por inquérito telefônico. Brasília: Ministério da Saúde; 2009. (Série G. Estatística e Informação em Saúde).

27. Secretaria de Gestão Estratégica e Participativa, Secretaria de Vigilância em Saúde, Ministério da Saúde. Vigitel Brasil 2009: vigilância de fatores de risco e proteção para doenças crônicas por inquérito telefônico. Brasília: Ministério da Saúde; 2010. (Série G. Estatística e Informação em Saúde).

28. Secretaria de Gestão Estratégica e Participativa, Secretaria de Vigilância em Saúde, Ministério da Saúde. Vigitel Brasil 2010: vigilância de fatores de risco e proteção para doenças crônicas por inquérito telefônico. Brasília: Ministério da Saúde, 2011. (Série G. Estatística e Informação em Saúde).

29. Secretaria de Vigilância em Saúde, Ministério da Saúde. Vigitel Brasil 2011: vigilância de fatores de risco e proteção para doenças crônicas por inquérito telefônico. Brasília: Ministério da Saúde; 2012. (Série G. Estatística e Informação em Saúde).

30. Departamento de Vigilância de Doenças e Agravos não Transmissíveis e Promoção de Saúde, Secretaria de Vigilância em Saúde, Ministério da Saúde. Vigitel Brasil 2012: vigilância de fatores de risco e proteção para doenças crônicas por inquérito telefônico. Brasília: Ministério da Saúde; 2013. 
31. Departamento de Vigilância de Doenças e Agravos não Transmissíveis e Promoção da Saúde, Secretaria de Vigilância em Saúde, Ministério da Saúde. Vigitel Brasil 2013: vigilância de fatores de risco e proteção para doenças crônicas por inquérito telefônico. Brasília: Ministério da Saúde; 2014.

32. Departamento de Vigilância de Doenças e Agravos não Transmissíveis e Promoção da Saúde, Secretaria de Vigilância em Saúde, Ministério da Saúde. Vigitel Brasil 2015: vigilância de fatores de risco e proteção para doenças crônicas por inquérito telefônico. Brasília: Ministério da Saúde; 2015.

33. Departamento de Vigilância de Doenças e Agravos não Transmissíveis e Promoção da Saúde, Secretaria de Vigilância em Saúde, Ministério da Saúde. Vigitel Brasil 2016: vigilância de fatores de risco e proteção para doenças crônicas por inquérito telefônico. Brasília: $\mathrm{Mi}$ nistério da Saúde; 2017.

34. Lwanga S, Lemeshow S. Sample size determination in health studies: a practical manual. Geneva: World Health Organization; 1991.

35. Ainsworth BE, Haskell WL, Whitt MC, Irwin ML, Swartz AM, Strath SJ, et al. Compendium of physical activity codes and MET intensities. Med Sci Sports Exerc 2000; 32(9 Suppl):S498-504.

36. Antunes JLF, Cardoso MRA. Using time series analysis in epidemiological studies. Epidemiol Serv Saúde 2015; 24:565-76.

37. Matsudo VK, Matsudo SM, Araujo TL, Andrade DR, Oliveira LC, Hallal PC. Time trends in physical activity in the state of São Paulo, Brazil: 2002-2008. Med Sci Sports Exerc 2010; 42:2231-6.

38. Ajzen I, Madden TJ. Prediction of goal-directed behavior: attitudes, intentions, and perceived behavioral control. J Exp Soc Psychol 1986; 22:453-74.

39. Dumith SC, Domingues MR, Gigante DP. Epidemiologia das atividades físicas praticadas no tempo de lazer por adultos do Sul do Brasil. Rev Bras Epidemiol 2009; 12:646-58.

40. Knuth AG, Hallal PC. Temporal trends in physical activity: a systematic review. J Phys Act Health 2009; 6:548-59.

41. Blair SN, Morris JN. Healthy hearts and the universal benefits of being physically active: physical activity and health. Ann Epidemiol 2009; 19:253-56.

42. Landi F, Abbatecola AM, Provinciali M, Corsonello A, Bustacchini S, Manigrasso L, et al. Moving against frailty: does physical activity matter? Biogerontology 2010; 11:537-45.

43. Mazini Filho ML, Zanella AL, Aidar FJ, Silva AMS, Salgueiro RS, Matos DG. Atividade física e envelhecimento humano: a busca pelo envelhecimento saudável. Revista Brasileira de Ciências do Envelhecimento Humano 2010; 7:97-106.
44. Vries NM, Ravensberg CD, Hobbelen JS, Olde RMG, Staal JB, Nijhuis-van SMW. Effects of physical exercise therapy on mobility, physical functioning, physical activity and quality of life in community-dwelling older adults with impaired mobility, physical disability and/or multi-morbidity: a meta-analysis. Ageing Res Rev 2012; 11:136-49.

45. Brovold T, Skelton DA, Bergland A. Older adults recently discharged from the hospital: effect of aerobic interval exercise on health-related quality of life, physical fitness, and physical activity. J Am Geriatr Soc 2013; 61:1580-5.

46. Taylor D. Physical activity is medicine for older adults. Postgrad Med J 2014; 90:26-32.

47. Craig CL, Russell SJ,Cameron C, Bauman A. Twenty-year trends in physical activity among Canadian adults. Can J Public Health 2004; 95:59-63.

48. Malta DC, Silva MMA, Albuquerque GM, Amorim RCA, Rodrigues GBA, Silva TS, et al. Política Nacional de Promoção da Saúde, descrição da implementação do eixo atividade física e práticas corporais, 2006 a 2014. Rev Bras Ativ Fis Saúde 2014; 19:286-99.

49. Gomes GAO, Kokubun E, Mieke GI, Ramos LR, Pratt M, Parra DC, et al. Characteristics of physical activity programs in the Brazilian primary health care system. Cad Saúde Pública 2014; 30:2155-68.

50. Guarda FRB, Silva RN, Araújo JLAC, Freitas MIF, Santos PM. Intervenção do profissional de educação física: formação, perfil e competências para atuar no Programa Academia da Saúde. Rev Pan-Amazônica Saúde 2014; 5:6374.

51. Departamento de Atenção Básica, Secretaria de Atenção à Saúde, Ministério da Saúde. Revista brasileira saúde da família. Brasília: $\mathrm{Mi}-$ nistério da Saúde; 2006.

52. Amorim TC, Knuth AG, Cruz DKA, Malta DC, Reis RS, Hallal PC. Descrição dos programas municipais de promoção da atividade física financiados pelo Ministério da Saúde. Rev Bras Ativ Fís Saúde 2013; 18:63-74.

53. Hallal PC, Tenório MC, Tassitano RM, Reis RS, Carvalho YM, Cruz DK, et al. Avaliação do programa de promoção da atividade física Academia da Cidade de Recife, Pernambuco, Brasil: percepções de usuários e não-usuários. Cad Saúde Pública 2010; 26:70-8.

54. Bernal RTI, Malta DC, Araújo TS, Silva NN. Inquérito por telefone: pesos de pós-estratificação para corrigir vícios de baixa cobertura em Rio Branco, AC. Rev Saúde Pública 2013; 47:316-25

55. Lima-Costa MF, Matos DL, Camargos VP, Macinko J. Tendências em dez anos das condições de saúde de idosos brasileiros: evidências da Pesquisa Nacional por Amostra de Domicílios (1998, 2003, 2008). Ciênc Saúde Coletiva 2011; 16:3689-96. 
56. Malta DC, Iser BPM, Sá NNB, Yokota RTC, Moura L, Claro RM, et al. Tendências temporais no consumo de tabaco nas capitais brasileiras, segundo dados do VIGITEL 2006 a 2011. Cad Saúde Pública 2013; 29:812-22.

57. Francisco PMSB, Barros MBA, Segri NJ, Alves MCGP, César CLG, Carandina L, et al. Comparação das estimativas de prevalência de indicadores de saúde no Município de Campinas, São Paulo, Brasil, nos anos de 2001/2002 (ISA-SP) e 2008/2009 (ISA-Camp). Cad Saúde Pública 2013; 29:1149-60.

58. Monteiro CA, Moura EC, Jaime PC, Lucca A, Florindo AA, Figueiredo ICR, et al. Monitoramento de fatores de risco para as doenças crônicas por entrevistas telefônicas. Rev Saúde Pública 2005; 39:47-57.
59. Moura EC, Morais Neto OL, Malta DC, Moura L, Silva NN, Bernal R, et al. Vigilância de Fatores de Risco para Doenças Crônicas por Inquérito Telefônico nas capitais dos 26 estados brasileiros e no Distrito Federal (2006). Rev Bras Epidemiol 2008; 11 Suppl 1:20-37.

60. Szolnokin G, Hoffmann D. Online, face-toface and telephone surveys - comparing different sampling methods in wine consumer research. Wine Economics and Policy 2013; 2:57-66. 


\section{Abstract}

This study aimed to analyze time trends in leisure-time physical activity in adults in Brazilian state capitals and the Federal District from 2006 to 2016. The study was based on data from the Risk and Protective Factors Surveillance System for Chronic Noncommunicable Diseases Through Telephone Interview (VIGITEL), collected by the Ministry of Health from 2006 to $2016(n=572,437)$. VIGITEL conducts interviews annually with more than 50,000 adults $(\geq$ 18 years) living in households with hardline telephones. The main relevant VIGITEL questions for this study deal with leisure-time physical activity. We estimated the annual percentage of leisuretime physical activity in the three months prior to the interview, in addition to sufficient levels of such activit ( $\geq 150$ minutes/week), available from 2009 to 2016 for the adult population as whole and according to sex, age, and schooling. There was an increase $(p<0.05)$ both in the percentage of leisure-time physical activity in the three months prior to the interview (from 44.0 to $53.6 \%$, or 0.97 percentage points per year) and in the percentage of individuals that achieved recommended levels of physical activity, from 30.3 to $37.6 \%$ (1.20 percentage points per year) from 2009 to 2016. The increases were more frequent in women, younger adults, and those with more schooling. There was an increase in physical activity in the majority of the situations studied. Although this increase reduces the differences between men and women, the differences increased between young people and older individuals and between those at the extremes of educational levels.

Exercise; Noncommunicable Diseases; Adult

\section{Resumen}

El objetivo de este estudio fue analizar la tendencia temporal de la práctica de actividad física durante el tiempo de ocio entre adultos, en las capitales brasileñas y Distrito Federal, entre 2006 y 2016. Se utilizaron datos del Sistema de Vigilancia de Factores de Riesgo y Protección para Enfermedades Crónicas No Transmisibles por Entrevista Telefónica (VIGITEL), recogidos por el Ministerio de la Salud, durante el período entre 2006 y $2016(n=572.437)$. VIGITEL entrevista anualmente a más de cincuenta mil individuos adultos (edad $\geq 18$ años) que residen en domicilios con teléfono fijo. Las principales preguntas de VIGITEL de interés en este estudio tratan sobre la práctica de actividad física durante el tiempo de ocio. Se estimó el porcentaje anual de realización de actividad física en el tiempo de ocio, durante los tres meses que antecedieron a la entrevista, además del referente a la práctica con nivel suficiente ( $\geq 150$ minutos/semana), disponible entre los años 2009 y 2016, para el conjunto de la población, según sexo, edad y escolaridad. Se verificó un aumento $(p<0,05)$, tanto en el porcentaje de práctica de actividad física durante el ocio, en los tres meses que antecedieron a la entrevista (de 44,0 a 53,6\%, $0,97$ puntos porcentuales ( $p p) / a \tilde{n} o$ ), en cuanto al porcentaje de individuos que alcanzaron las recomendaciones de práctica de actividad física: de 30,3 a 37,6\% (1,20pp/año) entre 2009 a 2016. Los aumentos fueron más frecuentes entre las mujeres, en los individuos dentro de las menores franjas de edad y entre aquellos con mayor escolaridad. Se verificó un aumento de los niveles de práctica de actividad física en la mayoría de las situaciones investigadas. Aunque ese aumento haya reducido las diferencias entre hombres y mujeres, se acentuaron las diferencias entre jóvenes y personas más viejas y entre aquellos en los niveles extremos de escolaridad.

Ejercicio; Enfermedades No Transmisibles; Adulto

Recebido em 04/Jul/2017

Versão final reapresentada em 14/Jun/2018

Aprovado em 14/Jun/2018 